\title{
The Analysis of the Error Statistics in a 5x40 Gbit/s Fibre Link with Hybrid Amplification
}

\author{
Anton Skidin, Alexey Redyuk, Olga Shtyrina, Michael Fedoruk \\ Institute of Computational Technologies, Novosibirsk, 6 Acad. Lavrentjev avenue, \\ 630090, Russia \\ Novosibirsk State University, Novosibirsk, 2 Pirogova street, 630090, Russia
}

Alex Shafarenko

University of Hertfordshire, Department of Computer Science, Hatfield, AL10 9AB, UK

\begin{abstract}
We quantify the error statistics and patterning effects in a 5x40 Gbit/s WDM RZ-OOK SMF/DCF fibre link using hybrid Raman/EDFA amplification. By extensive use of a numerical model, we determine how the error statistics change with the transmission distance. This knowledge is used as a basis for a constrained coding technique in order to improve the transmission error rate. We propose an adaptive constrained code for mitigation of the patterning effects and demonstrate that this approach can substantially reduce the bit error rate $(B E R)$ even for very large values of the channel $B E R\left(B E R>10^{-1}\right)$. The proposed technique can be used in combination with forward error correction schemes (FEC) to extend the range of channel $B E R$ s that an FEC scheme is effective over.
\end{abstract}

Keywords:

optical channels, signal processing, amplification, communication networks, constrained codes, coding theory

Email addresses: ask@skidin.org (Anton Skidin), alexey.redyuk@gmail.com (Alexey Redyuk), olya.shtyrina@gmail.com (Olga Shtyrina), mife@ict.nsc.ru (Michael Fedoruk), A.Shafarenko@herts.ac.uk (Alex Shafarenko) 


\section{Introduction}

Knowledge of the $B E R$ statistics is important for the development of forward error correction schemes, which can improve system performance when the transmission impairments cannot be eliminated by channel design. Such impairments can stem from a variety of physical phenomena. For instance, in some dispersion-managed systems at high channel rates (40 Gbit/s and more), the dispersion broadening of carrier pulses leads to bit overlapping. The bit overlapping causes the neighbouring pulses to interact, which leads to inter-symbol interference and patterning effects. It is notable that bit-overlapping has been observed in submarine systems already at channel rates as low as $10 \mathrm{Gbit} / \mathrm{s}$ [1]. The on-off-keying format (OOK) in high-bitrate transmission is limited by the effects of intra-channel four-wave-mixing (ICFWM), which generates "ghost" pulses [2]-[3]. This is an important example of patterning in optical channels [4], which in digital communication is seen as dependency of the single-bit error probability on the surrounding bits. As another example of the same, we cite the patterning effects caused by the saturation of a semiconductor optical amplifier gain (see $[5,6]$ ). In this paper we present one more system with nontrivial patterning effects: an SMF/DCF RZ-OOK WDM link with hybrid Raman/EDFA amplification at a channel rate of $40 \mathrm{Gbits} / \mathrm{s}$.

We have observed that when the bit-error rate (before the FEC) is high $(B E R>0.1)$, the error statistics exhibit strong patterning effects. It is well known that the performance of FEC codes degrades precipitously when the $B E R$ exceeds a certain value. In particular, at $B E R>0.1$ most of the conventional FEC codes (including convolutional and turbo codes) tend to break down. Increasing the redundancy does not help due to the fact that multiple errors in the (rather large under such conditions) checksum make the decoder corrupt some correct data bits with a probability as significant as that for a true error to be corrected by a checksum that is received with not too many errors. Therefore, even if advanced FEC schemes are employed, other measure for countering specific transmission impairments may have to be deployed before the FEC to ensure the latter operates within its effective range.

Constrained codes represent one such measure. They have already been proposed for suppression of specific channel effects (see e.g. [7]-[14]) in highbit-rate systems. The performance of the constrained codes for the mitigation

of patterning effects was examined in [15]. In this paper we present an 


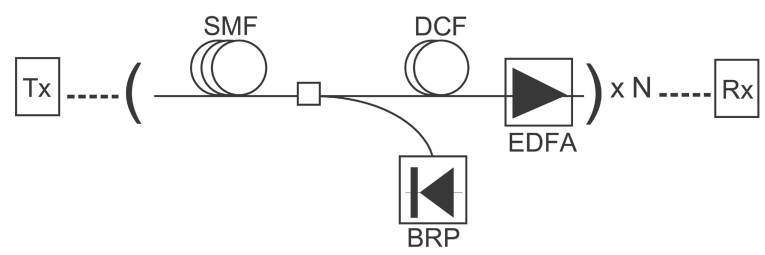

Figure 1: The scheme of the transmission line.

efficient adaptive constrained channel code as a more general solution to the problem discussed in [15].

\section{System configuration and error statistics}

Fig. 1 describes the transmission link used in our analysis. The unit cell of the link contains a two-step dispersion map with a span of single-mode fibre (SMF) $85 \mathrm{~km}$ long followed by a span of dispersion-compensated fibre (DCF). The accumulated dispersion of the SMF is overcompensated for by the DCF and the average dispersion of a single unit cell $<D>=-0.15$ $\mathrm{ps} / \mathrm{nm} / \mathrm{km}$. The basic parameters of the fibres are given in Table 1 . The amplification scheme is hybrid. A backward Raman amplifier and an erbiumdoped fiber amplifier (EDFA) with a noise figure of $4.5 \mathrm{~dB}$ and gain of 13.4 $\mathrm{dB}$ are combined to compensate for the losses in the SMF and DCF spans. The number of unit cells $N$ varies from 25 to 110. Further details of the transmission model can be found in [16]-[21].

We performed extensive numerical simulations of the 5x40 Gbit/s WDM RZ-OOK system. Pseudorandom binary sequences of 16384 bits were transmitted over the simulated line. The input pulses were unchirped Gaussian pulses with a duty cycle of $50 \%$ (12.5 ps pulse duration). At the receiver end a Gaussian optical bandpass filter (OBPF) and a third-order Butterworth filter with a cutoff frequency of $40 \mathrm{GHz}$ were used.

We have done in excess of 30 numerical experiments in total. The transmission distance varied in the range from $2500 \mathrm{~km}$ to $11000 \mathrm{~km}$. Each experiment consisted of 50 runs of a 5-channel WDM RZ-OOK transmission system with channels spaced out by $100 \mathrm{GHz}$. The total number of bits accumulated to determine the statistics discussed below were 4096000 per experiment. The total number of errors was counted while varying the decision level (DL) of the receiver. After each experiment the bit error rate was 
Table 1: Fibre parameters

\begin{tabular}{|c|c|c|}
\hline SMF & Attenuation at $1550 \mathrm{~nm}$ & $0.20 \mathrm{~dB} / \mathrm{km}$ \\
& Attenuation at $1455 \mathrm{~nm}$ & $0.25 \mathrm{~dB} / \mathrm{km}$ \\
& Effective Area & $80 \mu \mathrm{m}^{2}$ \\
& Dispersion & $17 \mathrm{ps} / \mathrm{nm} / \mathrm{km}$ \\
& Dispersion Slope & $0.07 \mathrm{ps} / \mathrm{nm}^{2} / \mathrm{km}$ \\
& Nonlinear index & $2.7 \cdot 10^{-20} \mathrm{~m}^{2} / \mathrm{W}$ \\
& $G_{\text {raman }} / A_{\text {eff }}(1455 \rightarrow 1550)$ & $0.421 / \mathrm{W} / \mathrm{km}$ \\
& Rayleigh backscattering pump & $7.05 \cdot 10^{-5} 1 / \mathrm{km}$ \\
& Rayleigh backscattering signal & $5.4 \cdot 10^{-5} 1 / \mathrm{km}$ \\
\hline DCF & Attenuation at 1550 nm & $0.65 \mathrm{~dB} / \mathrm{km}$ \\
& Effective Area & $19 \mu \mathrm{m}^{2}$ \\
& Dispersion & $-100 \mathrm{ps} / \mathrm{nm}^{2} / \mathrm{km}$ \\
& Dispersion Slope & $-0.41 \mathrm{ps} / \mathrm{nm}^{2} / \mathrm{km}$ \\
& Nonlinear index & $2.7 \cdot 10^{-20} \mathrm{~m}^{2} / \mathrm{W}$ \\
\hline
\end{tabular}

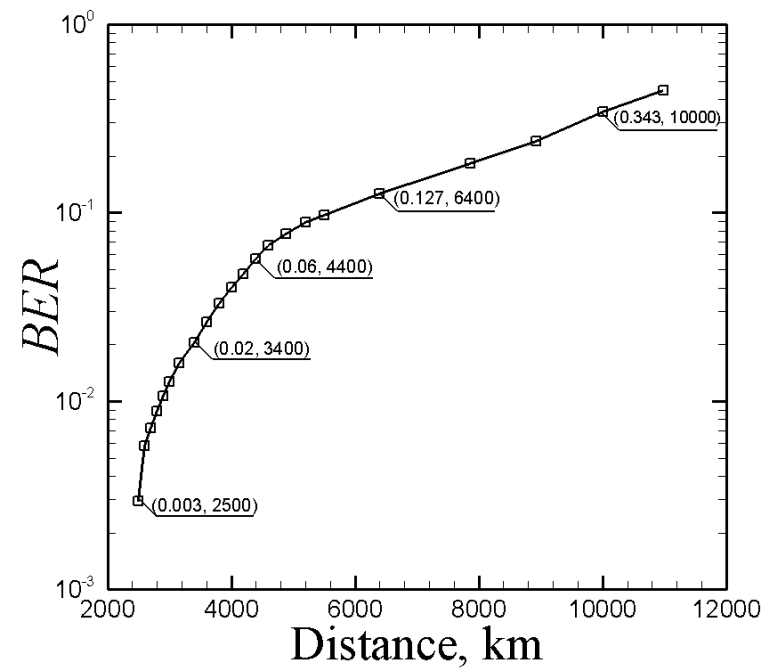

Figure 2: BER vs transmission distance. 


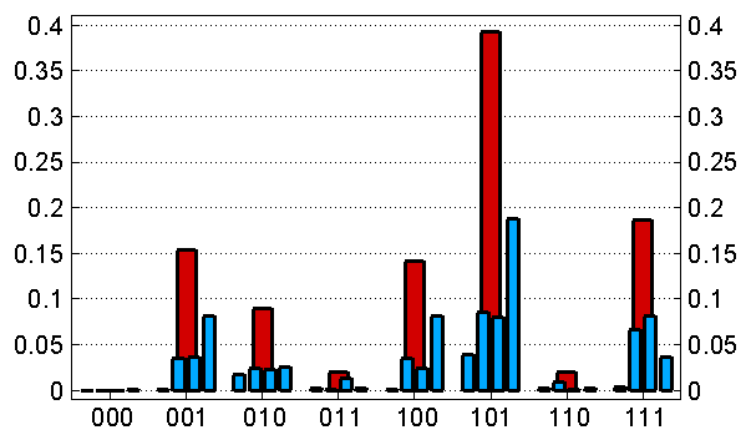

Figure 3: Error distribution past 4,500 km.

defined as

$$
B E R=\frac{N_{\text {err }}}{N_{\text {all }}}
$$

where $N_{\text {err }}$ is the number of error bits and $N_{\text {all }}$ is the number of all transmitted bits.

Figure 2 shows the directly calculated $B E R$ against the transmission distance. The largest deviation of $B E R$ value in each experiment is $3.83 \%$ (after 30 section). As expected, the bit-error rate rises with the distance due to a combination of nonlinear effects, chromatic dispersion and spontaneous emission noise injected by the amplifiers. As follows from figure 2 , the bit error rate becomes prohibitively large $(B E R>0.1)$ when the distance exceeds $5000 \mathrm{~km}$. At such distances for the system in question common FEC schemes would be expected to break down. However, the $B E R$ growth rate is decreasing as the distance increases, since some errors can be randomly corrected by the further signal distortion.

\section{Adaptive constrained coding}

Let us consider Table 2, in which the total numbers of errors are shown against the 32 quintuplets of neighbouring bits for several experiments. It is clear from this table that the error statistics change from experiment to experiment and still have an uneven distribution when the BER is large. Also it is clear that of all the basic quintuplets, some (e.g. 11011, 01011, 11010) are much worse than the rest.

The error distribution past the $4,500 \mathrm{~km}$ point is shown graphically in figure 3. The red bars represent the triplet error distribution and the blue 
ones the error distribution for quintuplets. For instance, blue bars that surround bar "101", from left to right correspond to quintuplets 01010, 01011, 11010 and 11011. Other bars should be interpreted in the same way. It can be shown that $18 \%$ of the errors are errors in quintuplet 11011, whose statistical weight is $1 / 32=3.125 \%$ (assuming the maximum entropy message). Figure 4 shows the difference between eye diagrams of detected signal for triplet 101 and 000. Due to distortion of the middle bit triplet 101 is a hard case for receiver. Optimal desicion level is highlighted by black line. Below we demonstrate that the error rate can be significantly reduced by exploiting the unevenness of the error statistics.

A common approach in a patterning situation is to apply channel preencoding in order to suppress some if not all the worst patterns. Let us consider a table block code $W=\left\{w_{i}\right\},|W|=2^{n}$ that achieves it by arranging all possible codewords in the ascending order of their a priori transmissionerror probabilities: $\forall i \in\left[0 . .2^{m}-1\right]: P\left(w_{i+1}\right) \geq P\left(w_{i}\right)$. The table $\mathrm{W}$ could then be used by an adaptive encoder/decoder.

Now that we have examined the quintuplet error statistics, let us build an adaptive code for quintuplets in the general case. Each sequence of $m$ bits has $m-4$ quintuplets. Denote as $q_{i}$ (a) the $i$ th quintuplet in a codeword a. If $\mathbf{a}=a_{0} a_{1} \ldots a_{m-1}$ is the binary representation of a length- $m$ codeword, then $q_{0}(\mathbf{a})=a_{0} a_{1} a_{2} a_{3} a_{4}, q_{1}(\mathbf{a})=a_{1} a_{2} a_{3} a_{4} a_{5}, \ldots, q_{k}(\mathbf{a})=a_{k} a_{k+1} a_{k+2} a_{k+3} a_{k+4}$ $(0 \leq k \leq m-5)$. For instance, codeword $\mathbf{a}=0101101$ has three quintuplets: $q_{0}(\mathbf{a})=01011, q_{1}(\mathbf{a})=10110$ and $q_{2}(\mathbf{a})=01101$. The codeword $a$ priori error probability can be determined as

$$
P\left(w_{i}\right)=1-\sum_{j=0}^{m-5}\left(1-R\left(q_{j}\left(w_{i}\right)\right),\right.
$$

where $R(p)$ is the a priori error probability of the quintuplet $p$.

Let us represent the source bitstream $S$ as a sequence of small blocks $s_{i}$ of length $n(n \leq m): S=\left(s_{1}, s_{2}, \ldots, s_{p}\right)$. Denote as $D=\left(d_{0}, d_{1}, \ldots, d_{p}\right)$ the encoded message, where $d_{i}$ is a length- $m$ data block. Now consider an adaptive block code $B(m, n)$ that maps the blocks $s_{i}$ onto the blocks $d_{i}$ as follows: $d_{i}=W\left(s_{i}\right), i=1 . . p$. This way, only the codewords with indices less than $2^{n}$ are used in the coding process. For decoding we need the table $W^{-1}(j), 0 \leq j<2^{m}: W^{-1}(r)=j$ if $W(j)=r$. Then the decoding is done by finding $s_{i}=W^{-1}\left(d_{i}\right), i=1$..p. This code has a redundancy of $R=\frac{m-n}{n}$. The error prevention power of the code depends solely on the smallness of $n$. 


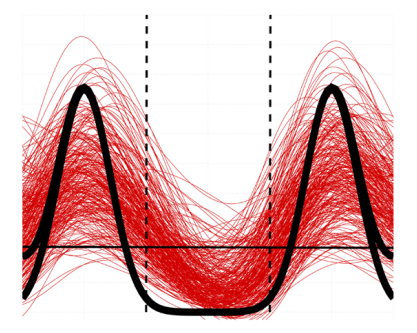

101

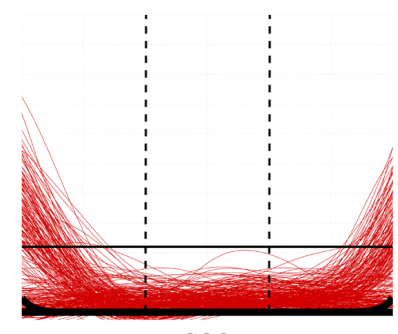

000

Figure 4: Comparison of the initial intensity waveforms and electrical eye diagrams of detected signal for triplets 101 and 000 after 4,500-km transmission.

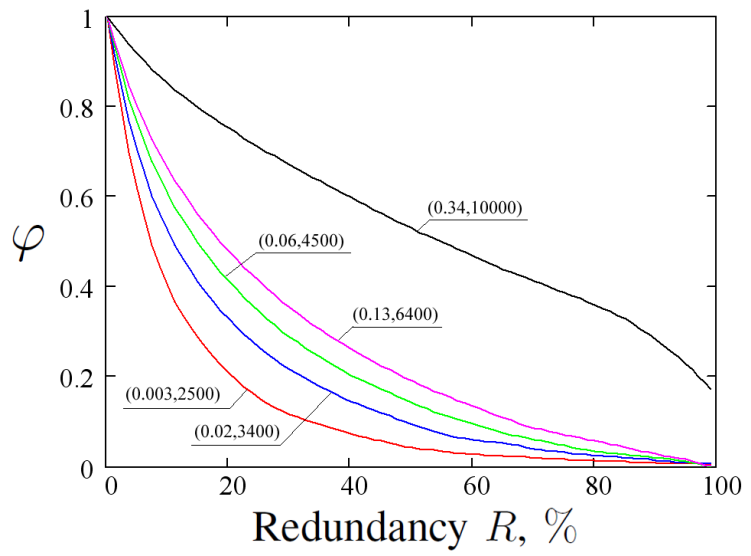

Figure 5: BER reduction ratio vs 24-bit adaptive code redundancy. 
Table 2: Total number of errors for 32 basic quintuplets

\begin{tabular}{|c|c|c|c|c|c|}
\hline $\begin{array}{c}\text { Distance, } \\
\text { km }\end{array}$ & 2500 & 3400 & 4500 & 6400 & 10000 \\
\hline $\mathbf{B E R}$ & 0.00296 & 0.0205 & 0.05989 & 0.1266 & 0.343 \\
\hline $\mathbf{0 0 0 0 0}$ & 0 & 0 & 0 & 6 & 7574 \\
\hline $\mathbf{0 0 0 0 1}$ & 0 & 0 & 0 & 124 & 26104 \\
\hline $\mathbf{0 0 0 1 0}$ & 0 & 10 & 245 & 1228 & 30955 \\
\hline $\mathbf{0 0 0 1 1}$ & 67 & 1441 & 8631 & 13612 & 67806 \\
\hline $\mathbf{0 0 1 0 0}$ & 50 & 882 & 4270 & 10754 & 80157 \\
\hline $\mathbf{0 0 1 0 1}$ & 78 & 1279 & 6051 & 13578 & 47279 \\
\hline $\mathbf{0 0 1 1 0}$ & 58 & 303 & 637 & 2464 & 48985 \\
\hline $\mathbf{0 0 1 1 1}$ & 1 & 31 & 250 & 2197 & 43531 \\
\hline $\mathbf{0 1 0 0 0}$ & 0 & 10 & 197 & 2684 & 18444 \\
\hline $\mathbf{0 1 0 0 1}$ & 81 & 1983 & 8724 & 25264 & 40064 \\
\hline $\mathbf{0 1 0 1 0}$ & 155 & 2284 & 9726 & 21502 & 31440 \\
\hline $\mathbf{0 1 0 1 1}$ & 704 & 5929 & 20892 & 49446 & 46298 \\
\hline $\mathbf{0 1 1 0 0}$ & 5 & 71 & 449 & 1505 & 66318 \\
\hline $\mathbf{0 1 1 0 1}$ & 86 & 542 & 2388 & 4707 & 35811 \\
\hline $\mathbf{0 1 1 1 0}$ & 4 & 76 & 746 & 11436 & 52307 \\
\hline $\mathbf{0 1 1 1 1}$ & 165 & 3119 & 16230 & 23542 & 35949 \\
\hline $\mathbf{1 0 0 0 0}$ & 0 & 0 & 3 & 274 & 18237 \\
\hline $\mathbf{1 0 0 0 1}$ & 0 & 21 & 226 & 3141 & 38507 \\
\hline $\mathbf{1 0 0 1 0}$ & 63 & 1915 & 8765 & 22616 & 36926 \\
\hline $\mathbf{1 0 0 1 1}$ & 672 & 6508 & 20029 & 34353 & 68275 \\
\hline $\mathbf{1 0 1 0 0}$ & 55 & 1072 & 5705 & 12796 & 62239 \\
\hline $\mathbf{1 0 1 0 1}$ & 120 & 1558 & 6150 & 12729 & 25329 \\
\hline $\mathbf{1 0 1 1 0}$ & 524 & 1915 & 3098 & 7347 & 48690 \\
\hline $\mathbf{1 0 1 1 1}$ & 64 & 360 & 603 & 2738 & 38269 \\
\hline $\mathbf{1 1 0 0 0}$ & 250 & 2711 & 5841 & 21140 & 42161 \\
\hline $\mathbf{1 1 0 0 1}$ & 1675 & 9538 & 19967 & 37046 & 81117 \\
\hline $\mathbf{1 1 0 1 0}$ & 812 & 6346 & 19788 & 51342 & 56466 \\
\hline $\mathbf{1 1 0 1 1}$ & 3637 & 18148 & 46061 & 84026 & 81422 \\
\hline $\mathbf{1 1 1 0 0}$ & 0 & 14 & 343 & 2497 & 43381 \\
\hline $\mathbf{1 1 1 0 1}$ & 8 & 130 & 522 & 4951 & 20970 \\
\hline $\mathbf{1 1 1 1 0}$ & 456 & 6773 & 19628 & 36370 & 50069 \\
\hline $\mathbf{1 1 1 1 1}$ & 2358 & 8999 & 8933 & 1126 & 13868 \\
\hline & & & & & \\
\hline
\end{tabular}




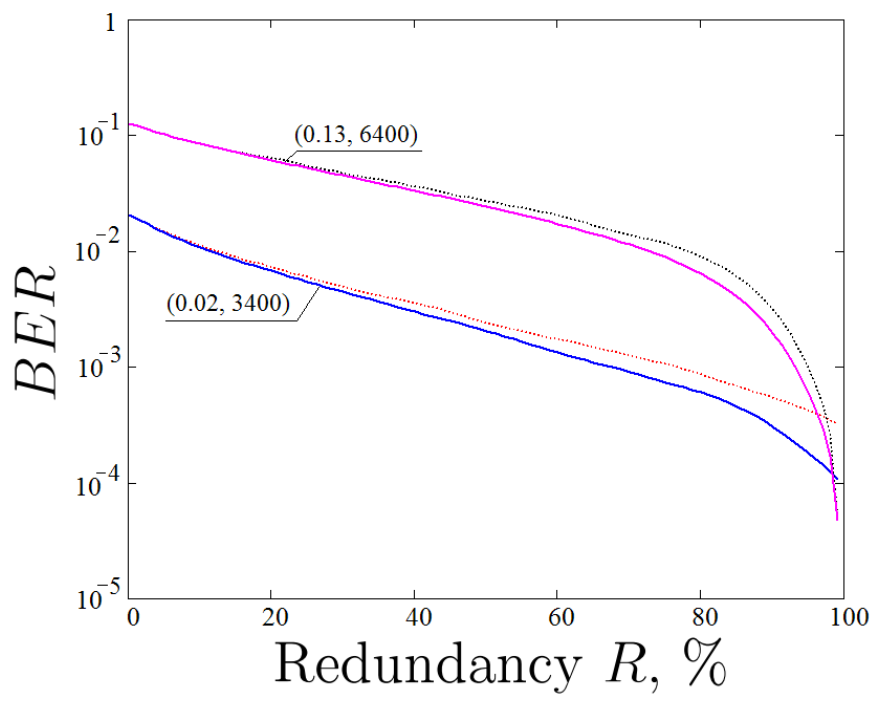

Figure 6: BER vs code redundancy for 16 and 24-bit block codes.

We have constructed the 16- and 24-bit block codes for our simulated transmission over the distances 2500, 3400, 4500, 6400 and $10000 \mathrm{~km}$. Figure 5 shows the observed reduction of the $B E R$. In the fugure, $\varphi$ is the bit error rate reduction ratio, $\varphi=\frac{B E R(R)}{B E R(R=0)}$; the pairs in brackets $(s, t)$ refer to the experiment parameters: $s$ is the BER value for uncoded signal, and $t$ is the number of sections. It should be noted that at the expense of only $20 \%$ of the bandwidth the BER can be reduced at least by a factor of 3 for smaller values $\left(B E R<10^{-2}\right)$ or at least by a factor of 2 for larger ones.

The code used above has a block length of 24 bits. It requires the mapping of $2^{24}$ codewords, each codeword is of 24 bits. The table $W$ requires $2^{24} \cdot 24$ bits, or 48 MBytes. Similarly, the table $W^{-1}$ requires 48 MBytes. The storage size of 100 MBytes is easily available today, and the adaptive encoding can be realized with little difficulty.

For comparison we examined the 16-bit block code as well. The performance data are given in figure 6 . The curves for the 16-bit code are dotted. Evidently 24 bits do not give a considerable improvement. Figure 6 shows that $B E R=0.13$ can be reduced to $B E R=10^{-3}$ using our adaptive code. This demonstrates that in some cases an adaptive constrained code can supplement the FEC-code with a gain in performance, since FEC does not work well at $B E R>10^{-1}$. 
Let us look into the special properties of constrained codes as compared to FEC-codes under the same conditions. First denote as $B E R^{\prime}$ the bit-error rate after the message has been decoded; similarly, denote as $B E R_{L}$ the largest value of $B E R$ for which the correction code still works satisfactorily. The latter can be found experimentally for any code, and is usually between $5 \cdot 10^{-2}$ and $10^{-1}$. Most advanced FEC can tolerate a $B E R$ of slightly more than $10^{-1}$; however they tend to be too complex for the transmission rates characteristic of optical communications. In our analysis we assume that at $B E R<B E R_{L}$, the FEC scheme achieves virtually error-free copy, and that above that threshold no amount of error correction would be effective. That is where the proposed adaptive constrained codes come into play. Even though they cannot correct the message, they can reduce the error rate prior to the FEC decoding stage. The error reduction should be based, as we suggested above, on the channel error statistics, which depend on its nonlinear properties ${ }^{1}$. To give an idea of the efficacy of our approach, figure 6 suggests that the error rate can be reduced up to $30 \%$ using a code with a small redundancy $\approx 5 \%$.

\section{Conclusions}

We have presented analysis of the error statistics and have quantified the patterning effects through extensive direct numerical modelling of a WDM RZ-OOK SMF/DCF fibre link operating at 40 Gbit/s per channel. We have demonstrated a BER improvement through adaptive channel encoding that reduces the incidence of undesirable patterns. This proves that channel preencoding can, indeed, be an effective method of improving the BER. The coding method presented here can be used in conjunction with specially designed transmission schemes based on ultra-long fiber lasers (see e.g. [22][24]) to enable FEC schemes to correct errors beyond their effective limits.

The work was funded by grant No 02.740.11.5129 of the Ministry of Education and Science of the Russian Federation.

\footnotetext{
${ }^{1}$ Note that those statistics can in turn be influenced by nonlinearity management that involves advanced distributed amplification schemes.
} 


\section{References}

[1] A. Pilipetskii, "Propagation Effects at High Bit Rates," Optical Fiber Communication Conference'06, OWJ7, 2006.

[2] R. J. Essiambre, B. Mikkelsen, and G. Raybon, "Intra-channel crossphase modulation and four-wave mixing in high-speed TDM systems," Electronics Letters, vol. 35(18), pp. 1576-1578, 1999.

[3] P. V. Mamyshev and N. A. Mamysheva, "Pulse-overlapped dispersionmanaged data transmission and intrachannel four-wave mixing," Optics Letters, vol. 24(21), pp. 1454-1456, 1999.

[4] Shapiro E. G., Fedoruk M. P., Turitsyn S. K., "Direct modelling of error statistics at $40 \mathrm{Gbit} / \mathrm{s}$ rate in SMF/DCF link with strong bit overlapping," Electronic Letters, vol. 40(22), pp. 1436-1437, 2004.

[5] F. Matera, A. Mecozzi, M. Settembre, I. Gabitov, H. Haunstein, and S. K. Turitsyn, "Theoretical evaluation of the noise growth and the system performance for a link constitued by a chain of $\mathrm{N}$ optical amplifiers with in-line filters," OFC'98 Technical Digest, WM23, pp. 202-204, 1998.

[6] M. Settembre, F. Matera, V. Hgele, I. Gabitov, A.W. Mattheus, S.K. Turitsyn, "Cascaded optical communication systems with in-line semiconductor optical amplifiers," J. Lightwave Technology, vol. 15 (6), pp. 962-967, 1997.

[7] A. H. Gnauck, A. Mecozzi, M. Shtaif, and J. Wiesenfeld, "Modulation Scheme for Tedons," U.S. Patent Application, \#20020126359, 2001.

[8] E. G. Shapiro, M. P. Fedoruk, S. K. Turitsyn, and A. Shafarenko, "Reduction of nonlinear intrachannel effects by channel asymmetry in transmission lines with strong bit overlapping," IEEE Photonics Technology Letters, vol. 15(10), pp. 1473-1475, Oct. 2003.

[9] B. Vasic, V. S. Rao, I. B. Djordjevic, R. K. Kostuk, and I. Gabitov, "Ghost-pulse reduction in 40-Gb/s systems using line coding," IEEE Photonics Technology Letters, vol. 16(7), pp. 1784-1786, Jul. 2004. 
[10] A. Shafarenko, K. S. Turitsyn, and S. K. Turitsyn, "Skewed coding for suppression of pattern-dependent errors," Proc. of 31st European Conference on Optical Communication (ECOC 2005), vol. 2, pp. 193-194, Glasgow, United Kingdom, Sept. 2005.

[11] I. Djordjevic, B. Vasic, V. Rao, "Rate $2 / 3$ modulation code for suppression of intrachannel nonlinear effects in high-speed optical transmission," IEEE Proc. Optoelectronics, vol. 153(2), pp. 87-92, 2006.

[12] A. Shafarenko, K. S. Turitsyn, S. K. Turitsyn, "Information-Theory Analysis of Skewed Coding for Suppression of Pattern-Dependent Errors in Digital Communications," IEEE Trans. Comm., vol. 55, no. 2, pp. 237-241, 2007.

[13] A. Shafarenko, M. Fedoruk, A. Skidin, S. Turitsyn, "Efficient weaklyconstrained codes for mitigation of patterning effects in digital communications," Proc. of International Conference on Ultra Modern Telecommunications (ICUMT 2009), Oct. 2009.

[14] I. Djordjevic, B. Vasic, V. Rao, "Constrained coding techniques for the suppression of intrachannel nonlinear effects in high-speed optical transmission," Journal of Lightwave Technology, vol. 24(1), pp. 411-419, January 2006.

[15] A. Shafarenko, A. Skidin, S.K. Turitsyn. "Weakly-constrained codes for suppression of patterning effects in digital communications," IEEE Transactions on Communications, vol. 58(10), pp. 2845-2854, 2010.

[16] S. K. Turitsyn, M. P. Fedoruk, O. V. Shtyrina, A. V. Yakasov, A. Shafarenko, S. R. Desbruslais, K. Reynolds, R. Webb, "Patterning effects in a WDM RZ-DBPSK SMF/DCF optical transmission at 40Gbit/s channel rate," Optics Communications, 277(2), 264-268 (2007)

[17] S. Namiki, Y. Emori, "Ultrabroad-band Raman amplifiers pumped and gain-equalized by wavelength-division-multiplexed high-power laser diodes," IEEE Journal of Selected Topics in Quantum Electronics, vol. 7(1), pp. 3-16, 2001.

[18] H. Kidorf, K. Rotwitt, M. Nissov, M. Ma, E. Rabarijaona, "Pump interactions in a 100-nm bandwidth Raman amplifier," IEEE Photonics Technology Letters, vol. 11(5), pp. 530-532, 1999. 
[19] L. Helczynski, A. Berntson, "Comparison of EDFA and bidirectionally pumped Raman amplifier in a 40-Gb/s RZ transmission system," IEEE Photonics Technology Letters, vol. 13(7), pp. 669-671, 2001.

[20] J. Ania-Castanon, "Quasi-lossless transmission using second-order Raman amplification and fibre Bragg gratings," Optics Express, vol. 12(19), pp. 4372-4377, 2004.

[21] J. Ania-Castanon, I. Nasieva, N. Kurukitkoson, S. K. Turitsyn, C. Borsier, and E. Pincemin, "Nonlinearity management in fibre transmission systems with hybrid amplification," Optics Communications, 233, pp. 353-357, 2004.

[22] J D Ania-Castanon, T. J. Ellingham, R. Ibbotson, X. Chen, L. Zhang, and S K Turitsyn, Ultralong Raman fiber lasers as virtually lossless optical media, Phys. Rev. Lett. 96, 023902 (2006)

[23] S. K. Turitsyn, J. D. Ania-Castanon, S. A. Babin, V. Karalekas, P. Harper, D. Churkin, S. I. Kablukov, A. E. El-Taher, E. V. Podivilov, and V. K. Mezentsev, "270-km Ultralong Raman Fiber Laser," Phys. Rev. Lett. 103, 133901 (2009)

[24] J. D. Ania-Castanon, V. Karalekas, P. Harper, and S. K. Turitsyn, Simultaneous Spatial and Spectral Transparency in Ultralong Fiber Lasers, Phys. Rev. Lett. 101, 123903 (2008) 